\title{
STUDIO IN VITRO DI ASSOCIAZIONI \\ ANTIBIOTICHE MEDIANTE CHECKERBOARD: \\ DIFFICOLTÀ TECNICHE RILEVATE CON CEPPI DI P.AERUGINOSA ISOLATI DA PAZIENTI CON FIBROSI CISTICA.
}

\author{
Guardo G.; ScrifignanoV.; Grassi P.; Grasso E.; Torrisi C.; \\ Sciuto C.; Mezzatesta M.L.; Stefani S.; Nicoletti G.; \\ Sciacca A.
}

Dipartimento di Microbiologia ,Laboratorio Azienda Policlinico, Broncopneumologia, Dipartimento Pediatria Università Catania

L'utilizzo delle associazioni antibiotiche è necessario nelle riacutizzazioni di infezioni croniche dei pazienti con fibrosi cistica (FC) e indispensabile in presenza di ceppi batterici multi-resistenti.

Scopo del nostro lavoro è stato quello di saggiare in vitro su micropiastra - con il metodo di checkerboard - l'attività di alcune associazioni antibiotiche verso n. 15 ceppi di Pseudomonas aeruginosa isolati da pazienti con FC. Sono state saggiate le seguenti combinazioni antibiotiche: tobramicina + ciprofloxacina, tobramicina + piperacillina e piperacillina + ciprofloxacina.

Contemporaneamente veniva eseguito il test di associazione su piastra di Muller-Hinton con metodica di Kirby Bauer al fine di confrontare i due metodi.

I risultati da noi ottenuti hanno messo in evidenza sinergia tra cip /pip nel 50\% dei ceppi saggiati , tra tobr/pip nel 37,5\%, tra cip/tobr nel $25 \%$ e un sinergismo parziale rispettivamente del $25 \%$ e $37,5 \%$ tra cip/pip e tob/cip. Indifferenza è stata riscontrata tra tob/pip nel $50 \%$ degli isolati.

Risultati sovrapponibili sono stati ottenuti utilizzando il metodo di Kirby Bauer.

L'esecuzione delle prove di sensibilità con metodica di checkerboard verso gli isolati non fermentanti ha messo in evidenza alcune difficoltà tecniche:

alcuni saggi eseguiti su due ceppi mucoidi di P.aeruginosa richiedevano tempi di incubazione superiori di almeno $12 \mathrm{~h}$, rispetto a quelli suggeriti;

due ceppi di P.aeruginosa mucoidi non evidenziavano alcuna crescita anche dopo $48 \mathrm{~h}$, pur essendo vitali nella conta dell'inoculo in piastra; in questi casi l'aumento dell'inoculo fino a $10^{6}$ consentiva di effettuare la lettura del saggio;

Abbiamo migliorato la lettura dei tests ponendo in termosta-

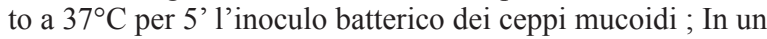
caso è stato necessario abbassare la temperatura di incubazione a $30^{\circ} \mathrm{C}$.

Le nostre conclusioni sono che sarebbe necessario rivedere le metodiche per lo studio delle associazioni, tanto utilizzate nel caso di infezioni in questo gruppo di pazienti. 